Revue des patrimoines

\title{
Retour sur un patrimoine parisien méconnu : les espaces de transmission du savoir à l'époque moderne (I). De la maison à l'amphithéâtre
}

\section{Christian Hottin}

\section{OpenEdition}

\section{Journals}

Édition électronique

URL : http://journals.openedition.org/insitu/3777

DOI : 10.4000/insitu.3777

ISSN : 1630-7305

Éditeur

Ministère de la culture

Référence électronique

Christian Hottin, « Retour sur un patrimoine parisien méconnu : les espaces de transmission du savoir à l'époque moderne (I). De la maison à l'amphithéâtre », In Situ [En ligne], 10 | 2009, mis en ligne le 19 mai 2009, consulté le 19 avril 2019. URL : http://journals.openedition.org/insitu/3777 ; DOI : 10.4000/ insitu. 3777

Ce document a été généré automatiquement le 19 avril 2019.

\section{(c) (i) ()}

In Situ Revues des patrimoines est mis à disposition selon les termes de la licence Creative Commons Attribution - Pas d'Utilisation Commerciale - Pas de Modification 4.0 International. 


\title{
Retour sur un patrimoine parisien méconnu: les espaces de transmission du savoir à l'époque moderne (I). De la maison à l'amphithéâtre
}

\author{
Christian Hottin
}

Le bâtiment du collège des Bernardins, restauré et officiellement inauguré lors de la visite du pape Benoît XVI le 4 septembre 2008, a connu une histoire que l'on peut voir comme une métaphore tout à fait emblématique du sort réservé au patrimoine immobilier des établissements d'enseignement de l'ancienne Université de Paris. Conservant des vestiges importants, visible depuis la rue, il n'en était pas moins totalement inconnu de tous ou presque, préservé de la destruction depuis deux siècles par une affectation utilitaire (une caserne de pompiers) qui rendait pratiquement impossible son appropriation en tant qu'objet patrimonial. Particulièrement riche, qualitativement mais aussi quantitativement, le patrimoine hérité de l'ancienne Université de Paris est à cette image : peu connu, peu visible, largement sous-estimé. Bien souvent, y compris pour ses défenseurs, l'attention focalisée sur les destructions de la Révolution et du XIX ${ }^{\mathrm{e}}$ siècle (vain martyrologe toujours réécrit avec délectation) empêche de voir le nombre et la diversité des édifices conservés. La comparaison, presque toujours implicite, avec le sort fait au patrimoine bâti d'autres universités européennes anciennes paraît décourager la comparaison et interdit pour ainsi dire de tenter de dresser l'inventaire de ce qui n'apparaît en définitive que comme des vestiges. Ils sont pourtant impressionnants et méritent qu'on s'y arrête. Ainsi, pour s'en tenir au cas des collèges, et sans compter les édifices qui jouissent déjà d'un prestige architectural reconnu (la Sorbonne, le collège des Quatre-Nations, le Collège de France), on peut dénombrer douze collèges et trois séminaires de l'ancienne Université qui subsistent, au moins partiellement, dans le Ve arrondissement de Paris ${ }^{1 .}$ Sans entrer dans le détail d'une recherche sur la causalité des phénomènes, gageons que les bouleversements sociaux et politiques survenus depuis la Révolution et qui ont trouvé leur traduction concrète sur le territoire de l'ancienne 
Université dans les innombrables mutations de propriété foncière et changements d'affectation fonctionnelle des édifices ne facilitent pas l'identification de ces immeubles avec une catégorie de patrimoine. Pour ceux qui sont toujours dévolus à l'enseignement, les transformations, sur deux siècles, des méthodes et moyens de transmission des connaissances, tout autant que les réécritures constantes de la physionomie architecturale des bâtiments, ne permettent pas non plus une approche globale: nombreuses sont les monographies, mais rares sont celles qui esquissent des comparaisons.

2 Laissant ici de côté la question des collèges, très complexe car ceux-ci étaient tout à la fois des lieux de vie et des lieux d'enseignement, où, concrètement, vie collective et enseignement n'étaient pas distincts, on a suivi une autre problématique, qui permette une approche comparée de plusieurs édifices - pour beaucoup conservés - constitutifs du patrimoine architectural de l'ancien Quartier latin. Comment, tout au long de l'époque moderne, d'abord à travers des solutions architecturales modestes (telles que les réappropriations) puis par la construction d'édifices de plus en plus complexes, sont apparus et se sont développés des espaces spécialement dédiés à l'élaboration et à la transmission du discours scientifique? Pour aborder ce sujet, le problème de l'implantation et de l'évolution des amphithéâtres est bien entendu essentiel. Mais on ne saurait oublier que l'implantation progressive de ce nouveau dispositif d'enseignement s'est faite dans un paysage architectural marqué par l'existence de quelques édifices spécialement conçus pour abriter les facultés : à ce titre s'impose une présentation d'un bâtiment tel que celui de l'ancienne faculté de médecine. Lorsque sont construits à Paris les premiers amphithéâtres, qui portent la marque des réalisations italiennes, cette institution est engagée dans une lutte appelée à durer près de deux siècles et qui va opposer les médecins aux chirurgiens : la compétition institutionnelle se traduit - entre autres - sur le plan architectural, à travers les projets de constructions élaborés successivement pour les deux établissements. Au fil des décennies, tandis que se perfectionnent les dispositifs matériels de transmission des connaissances, les amphithéâtres apparaissent également comme les espaces privilégiés de représentation de la communauté, notamment à travers le choix des décors.

\section{Écoles et facultés : de la demeure particulière à l'espace spécifique (1450-1750)}

\section{De la maison particulière à l'espace spécifique}

3 Une fois sorti des cloîtres des cathédrales ou des abbayes, l'enseignement se répand dans la ville et se glisse dans le tissu urbain; les premiers lieux d'enseignement sont des maisons particulières, que rien ne distingue des autres. Seule la qualité de leur locataire leur confère une spécificité, puisqu'elles ont été louées par un maitre pour que ce dernier $\mathrm{y}$ loge et $\mathrm{y}$ dispense son enseignement. On retrouve là une similitude entre l'institution universitaire et les autres guildes ou corporations: le maitre recherche une maison comportant une salle de belles dimensions et quelques annexes, exactement comme un artisan s'assure une échoppe ou un chantier en adéquation avec son activité ${ }^{2}$. L'aménagement reste sommaire : à Bologne, au XIV ${ }^{e}$ siècle, le local de Giovanni d'Andrea se compose simplement de chaises sans dossiers, d'une estrade et de pupitres pour les élèves $^{3}$. Les fondations les plus prestigieuses n'échappent pas à la règle; ainsi, en 1426, 
l'Université de Louvain achète une maison qui devient le siège de sa faculté des arts et le restera jusqu'en 1797, tandis que le Carolinum de Prague, fondé en 1366, est lui aussi installé dans la demeure d'un simple particulier ${ }^{4}$. Ce processus d'investissement de l'espace urbain ordinaire par l'institution enseignante va de pair avec un autre, également caractéristique de l'exercice de nombreux métiers au Moyen Âge. Il s'agit du regroupement et de la concentration dans un même secteur de la ville des personnes exerçant une activité semblable. Ce qui est manifeste à Paris avec la rue du Fouarre (pour les artiens) ou le Clos-Bruneau (pour les juristes) se rencontre dans les autres grandes villes universitaires, puisque les médecins investissent à Bologne la via della Porta nuova, tandis que les légistes ont leurs quartiers via delle Scuole ${ }^{5}$.

4 Au bas Moyen Âge, deux faits marquants déterminent le véritable point de départ de l'histoire des lieux d'enseignement. D'une part, les facultés qui jusqu'alors se contentaient de tenir leurs assemblées dans des églises acquièrent des locaux indépendants; d'autre part, sous l'impulsion des pouvoirs publics ou du fait des seuls universitaires, ces locaux tendent à devenir des lieux d'enseignement et non plus seulement de rassemblement. Telles sont les "grandes écoles » de Caen, bâties en 1436, et qui regroupent en un même lieu les arts et le droit (au rez-de-chaussée), la théologie et la médecine (au premier étage) et une bibliothèque commune (au deuxième étage $)^{6}$. De même, à Poitiers, la Ville s'érige en maître d'ouvrage en 1445 et 1466 pour doter sa jeune université de locaux indépendants ${ }^{7}$. À Paris, cependant, le mouvement se fait en ordre dispersé, les différentes facultés élisant domicile en divers lieux de la Montagne Sainte-Geneviève.

\section{Artiens et théologiens, juristes et médecins}

5 Konrad Rückbrod a reconstitué, plans à l'appui, l'emplacement successif des différentes facultés parisiennes, mettant ainsi en évidence les distinctions qui s'opèrent entre lieux d'enseignement établis chez des particuliers, sièges des assemblées des facultés et pour finir, véritables écoles ${ }^{8}$. La faculté des arts se fixe très tôt dans les parages de la rue du Fouarre, avec l'église Saint-Julien le Pauvre comme lieu d'assemblée, et la Sorbonne s'impose comme le principal centre des études théologiques, en même temps que lieu privilégié de rassemblement pour cette communauté. Si les juristes investissent le ClosBruneau dès le XIII ${ }^{e}$ siècle, les étudiants en médecine semblent avoir occupé plusieurs sites avant de s'établir : vers 1200 les études de médecine avaient lieu sur la rive droite, non loin du Louvre, mais un siècle plus tard elles avaient gagné la rive gauche, face à l'hôtel-Dieu de la Citég. Pour ces deux facultés, vers 1300, il semble que le lieu d'assemblée - et non le siège des études - ait été l'église des Mathurins, qui est aussi le siège de l'Université.

6 À la fin du Moyen Âge, les maîtres de la faculté des arts se répartissent dans les différents collèges, tandis que la Sorbonne voit confirmée sa position éminente dans la théologie. Si cette dernière faculté dispose au $\mathrm{XVI}^{e}$ siècle d'un siège indépendant, il paraît tellement modeste qu'on juge improbable son utilisation pour des délibérations générales ${ }^{10}$, lesquelles, comme on l'a vu, se tiennent en Sorbonne dès 1544. En revanche, pour le droit et la médecine, on assiste au regroupement en un même endroit des espaces de rassemblement et d'enseignement.

7 La configuration des «écoles de décret " demeure toutefois mal connue. Selon Simone Roux $^{11}$, elles étaient faites de "deux corps de logis, l'un devant et l'autre derrière, une cour au milieu et un petit jardin derrière ». D'après d'autres indications ${ }^{12}$, elles auraient 
été en fait constituées de deux édifices distincts, situés à quelque distance l'un de l'autre dans le Clos-Bruneau, et dénommés "grandes écoles », bâtis en 1486, et " petites écoles ». Aménagées de manière assez rudimentaires, les grandes écoles comportent deux salles superposées, celle du rez-de-chaussée destinée aux cours, celle de l'étage réservée aux thèses

Figure 1

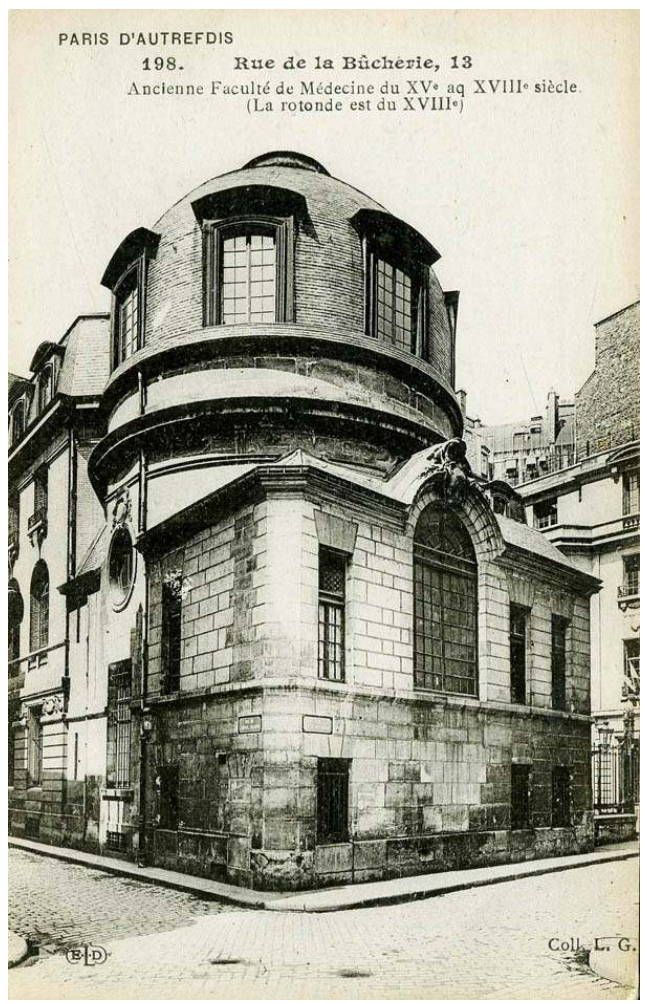

L'ancienne faculté de médecine de l'Université de Paris après sa restauration. Elle abrite alors le siège de l'association des étudiants de l'Université. Carte postale ancienne. Éditions E.L.D., collection L.G., Imp. E. Le Deley, Paris. (collection particulière) 


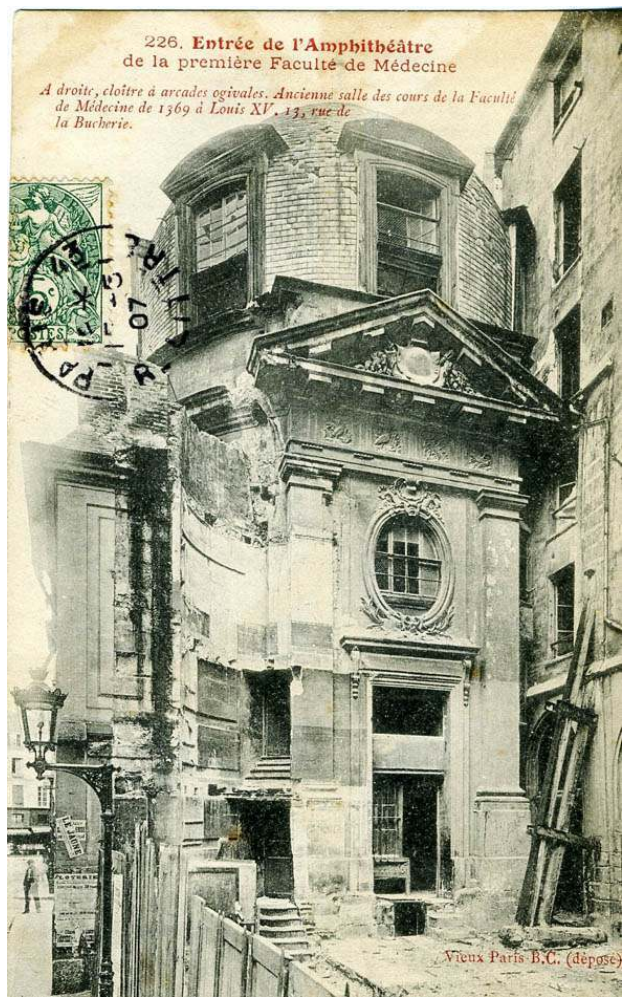

L'ancienne faculté de médecine de l'Université de Paris en cours de restauration. Carte postale ancienne. Collection Vieux Paris B.C. $n^{\circ}$ 226. (collection particulière).

8 La physionomie de la faculté de médecine est bien mieux connue ${ }^{13}$. Désaffectés, abandonnés, dépecés et pour finir lourdement restaurés au siècle dernier (fig. $\mathbf{n}^{\circ} \mathbf{1}, \mathbf{n}^{\circ} \mathbf{2}$ ), ses bâtiments subsistent pour l'essentiel ${ }^{14}$. Le témoignage archéologique que constituent ses murs est complété par le recueil des Commentaires de la faculté, conservé à la BIUM : par la mise en œuvre de ces sources on peut dessiner le contour d'un bâtiment universitaire, à la fois lieu d'étude et de manifestations collectives, profondément différent des collèges de la faculté des arts. Son histoire se distingue de celle des collèges par un trait qui n'est pas proprement architectural, mais qui conditionne tout processus de construction : tout au long de son existence, les moyens financiers de la faculté ont été particulièrement modestes ; contrairement à bien des collèges, elle ne bénéficie pas d'une dotation initiale et ses revenus sont très limités. En règle générale, ses projets de location, d'acquisition ou de construction reposent sur ses fonds propres. C'est le cas pour la première maison, acquise en 1369 « rue de la Buscherie, tirant la rue aux Rats », et qui, un siècle durant, fait seulement office d'entrepôt pour la très pauvre bibliothèque de l'institution. En 1454, un don de trente écus d'or de Jacques Despars, médecin de Philippe le Bon et de Charles VII, permet enfin d'envisager l'édification d'un bâtiment neuf. Pour y parvenir, il faut toutefois louer, pour 8 l. en 1469, puis acheter, 300 l. en 1470, une maison voisine aux chartreux. La faculté, exsangue, ne peut entreprendre les travaux que trois ans plus tard ${ }^{15}$. 


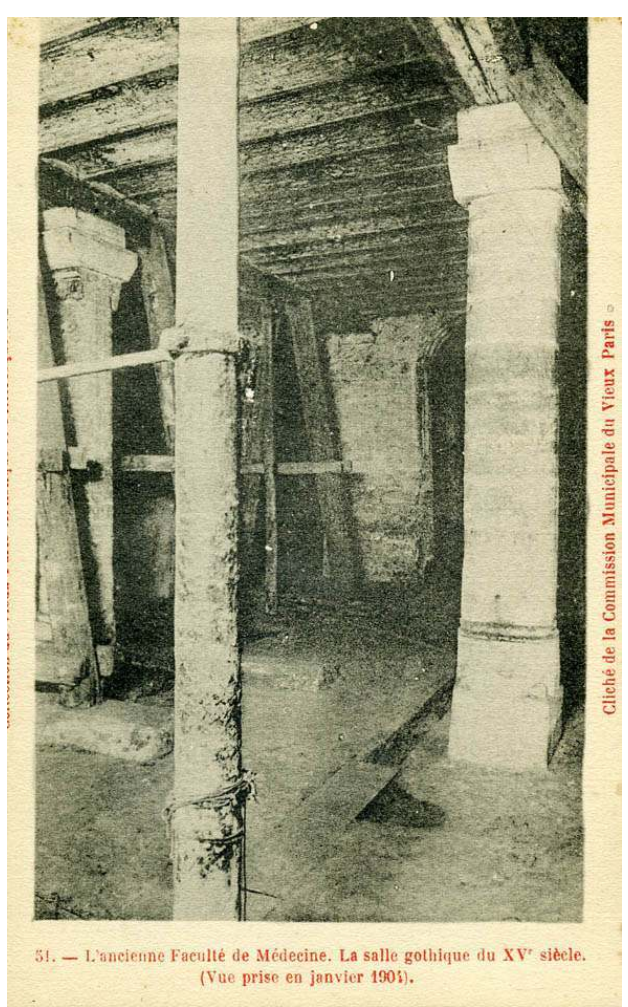

L'ancienne faculté de médecine de l'Université de Paris. La salle gothique du XVe siècle, vue prise en 1904. Carte postale ancienne. Cliché de la commission du Vieux Paris. Collection du Vieux Paris Artistique et Pittoresque $188, n^{\circ}$ 51. (collection particulière).

9 À cette pauvreté de moyens, criante quand on la compare aux revenus des grands collèges de l'Université, répond la modestie de l'édifice. Achevé seulement en 1481, il se présentait sous la forme d'un quadrilatère de 45 pieds de long sur 30 de large et haut de deux étages, sans compter le grenier. Une petite habitation, élevée en 1491 et d'abord réservée aux bedeaux, est transformée en chapelle en 1501, mais en 1528 un nouveau lieu de culte vient la remplacer au même endroit. La disposition intérieure est d'une grande simplicité. Le rez-de-chaussée, grande salle où deux piliers reçoivent seuls toute la poutraison, est affecté aux "écoles inférieures »: c'est le principal lieu d'enseignement et la salle de soutenance des thèses (fig. $\mathbf{n}^{\circ} \mathbf{3}$ ). Outre les pupitres des bacheliers, on y trouve deux chaires de hauteur différente, celle du maître et celle du bachelier soutenant la dispute. À l'étage principal, "les écoles supérieures", ou aula major, constituent l'espace d'assemblée des docteurs de la faculté ${ }^{16 .}$ Presque rien, on le voit, dans ces dispositions sommaires, qui renvoie spécifiquement à l'exercice de la médecine: le temps des amphithéâtres n'est pas encore venu; toutefois, en 1508, un petit jardin botanique est aménagé devant la salle des actes, afin, parait-t-il, d'éviter aux étudiants «les longues excursions botaniques dans la plaine de Gentilly ${ }^{17}$. 


\section{Un nouveau lieu pour un nouvel enseignement :

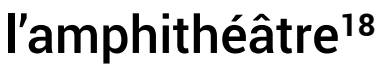

\section{L'antériorité italienne}

La redécouverte de l'amphithéâtre, en tant que dispositif architectural de transmission du savoir, est indissociable du développement de l'anatomie et de la pratique de la dissection. Simultanément, cette discipline et cette pratique accompagnent l'avènement du cours magistral comme méthode d'enseignement: il ne s'agit plus, comme dans la tradition médiévale, de commentaires ou de disputes, mais d'explications et de démonstrations; une bonne visibilité de l'action pratiquée est en outre indispensable pour la compréhension du discours du maitre ${ }^{19}$. Reste que cet enseignement peine à s'imposer, en butte qu'il est aux réticences de l'Église. Si une dissection publique a bien lieu en 1505 dans l'hôtel de Nesles, le fait demeure exceptionnel, et c'est la clandestinité qui prévaut ${ }^{20}$. En dehors de France, on construit les premiers amphithéâtres d'anatomie, d'abord provisoires, tels ceux imaginés par Vésale à Bologne et Padoue ${ }^{21}$ puis bâtis pour durer, toujours à Padoue en 1594. Si le type architectural établi à Padoue (amphithéâtre circulaire et forte pente des gradins disposés de manière concentrique) est appelé à perdurer, l'édifice italien se distingue de ceux élevés au siècle suivant par deux « défauts » de conception : aucune place assise n'est prévue sur les dix gradins entourant la table de dissection, et la pièce ne comporte pas d'ouvertures ${ }^{22}$. Peu d'années après, cette forme architecturale nouvelle gagne les faveurs des médecins et des chirurgiens parisiens, au point de faire du premier amphithéâtre à bâtir sur les rives de la Seine une des manifestations de la rivalité qui oppose les deux professions.

\section{Médecins et chirurgiens : la course à l'amphithéâtre}

11 Face à la faculté de médecine, détentrice historique et académique de l'art de soigner, la corporation des chirurgiens de "robe longue ${ }^{23}$ n'a eu de cesse d'obtenir le droit d'enseigner. Dès 1544, la faveur royale leur octroie des privilèges fiscaux identiques à ceux des universitaires, avant que la papauté ne leur accorde en 1579, sous certaines réserves, la licence d'enseigner ${ }^{24}$. Dès lors, les chirurgiens dispensent dans une salle voisine de l'église Saint-Côme des leçons publiques et parent ce modeste édicule, cette " sallette ", du titre de " collège royal de chirurgie ». Ils semblent toucher au but quand les médecins, jouant les chirurgiens « de robe courte » contre ceux de " robe longue », les contraignent à faire soumission à la faculté en 1596. C'est dans ce contexte conflictuel que s'inscrit la construction des premiers amphithéâtres anatomiques parisiens.

En 1608, Henri IV accorde au chirurgien Séverin Pineau 6000 livres pour l'instruction de dix aspirants, puis la mise à disposition, en 1609, d'une salle dans le collège d'Inville, voisin de l'église Saint-Côme. Presque au même moment, André du Laurens, premier médecin du roi, obtient qu'Henri IV, par l'édit du 17 juin 1608, enjoigne la faculté « de bastir un théâtre anatomique dans une place appartenant autrefois au collège de Karesbec ${ }^{25}$ ». D'emblée, les médecins manquent cette occasion : ils entrent en résistance contre l'édit, refusant de quitter la rue de la Bûcherie et de suivre du Laurens, qui est montpelliérain. Pendant que Riolan le Jeune prend la tête de la fronde médicale et engage une bataille qui va durer presque dix ans, les chirurgiens s'établissent : ils achètent aux 
marguilliers de Saint-Côme un terrain plus vaste que leur « sallette » et y font rapidement bâtir un petit amphithéâtre carré de trois toises et demie de côté, complété par une galerie. Inaugurée en novembre 1616, cette salle, d'une capacité de 100 à 200 personnes, est élevée grâce aux deniers de la communauté, chaque chirurgien devant apporter un minimum de 300 livres tournois à l'entreprise ${ }^{26 .}$ Pendant ce temps, les médecins bataillent pour voir s'élever leur amphithéâtre au sein de la faculté et non ailleurs... L'arrêt du Parlement du 18 septembre 1617 leur donne gain de cause, les travaux commencent le mois suivant, et Riolan inaugure en 1620 l'amphithéâtre qui portera son nom.

Après cette première manche indécise, la supériorité des chirurgiens se manifeste avec plus d'éclat lors de la construction du deuxième amphithéâtre de chaque communauté. En effet, quoique très attachés à leur maison de la rue de Bûcherie "tirant la rue aux Rats ", les médecins ont conscience des insuffisances de leur local et des inconvénients irrémédiables de sa situation, au cœur d'un quartier peu attrayant et sujet plus que d'autres aux inondations. Dès 1638, ils demandent aux médecins du roi, Bouvard et Cousinot, d'intercéder en leur faveur pour obtenir la jouissance du collège de Bourgogne ${ }^{27}$ , qui leur est refusée. L'année suivante, nouvelle tentative, et nouvel échec, pour demander l'attribution de l'hôtel de Nesmond, sis quai de la Tournelle ${ }^{28}$. Sous Louis XIII et Louis XIV, quelques donations leur permettent tout au plus des travaux d'embellissement dans une demeure toujours plus décrépite... Cependant, les chirurgiens sont devenus une florissante communauté forte de 300 membres, et ils jugent trop modeste et trop exigu leur amphithéâtre carré. En juin 1691, ils obtiennent des cordeliers voisins un terrain de 11 toises de façade sur la rue et sept et demie de profondeur, contre une rente annuelle de 600 livres $^{29}$. (fig. $n^{\circ} 4, n^{\circ} 5$ ) 
Figure 4

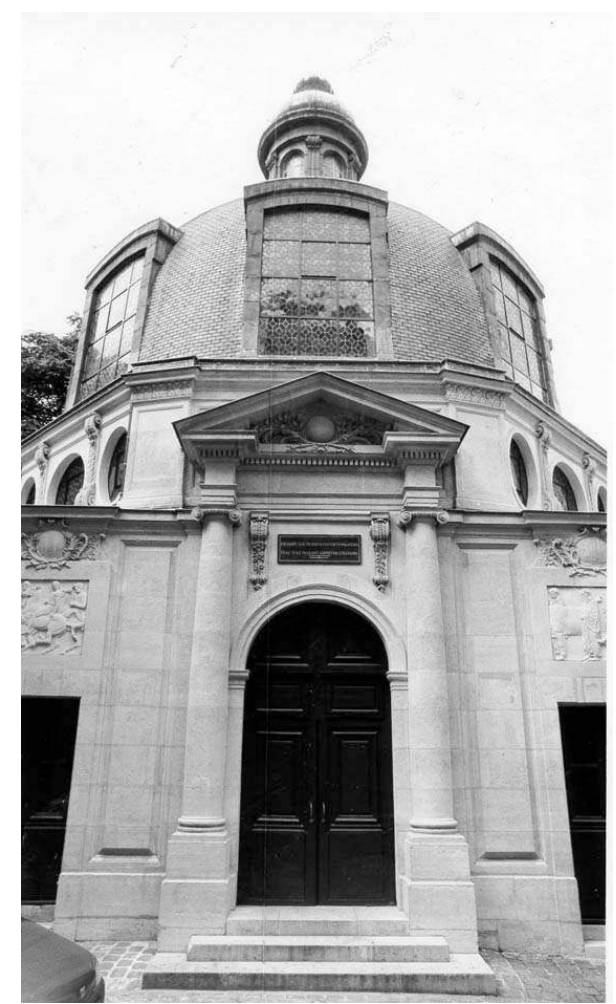

L'amphithéâtre de l'École de chirurgie, rue de l'École de médecine. La façade principale. Photo. JeanChristophe Doërr.

(c) Jean-Christophe Doërr. 


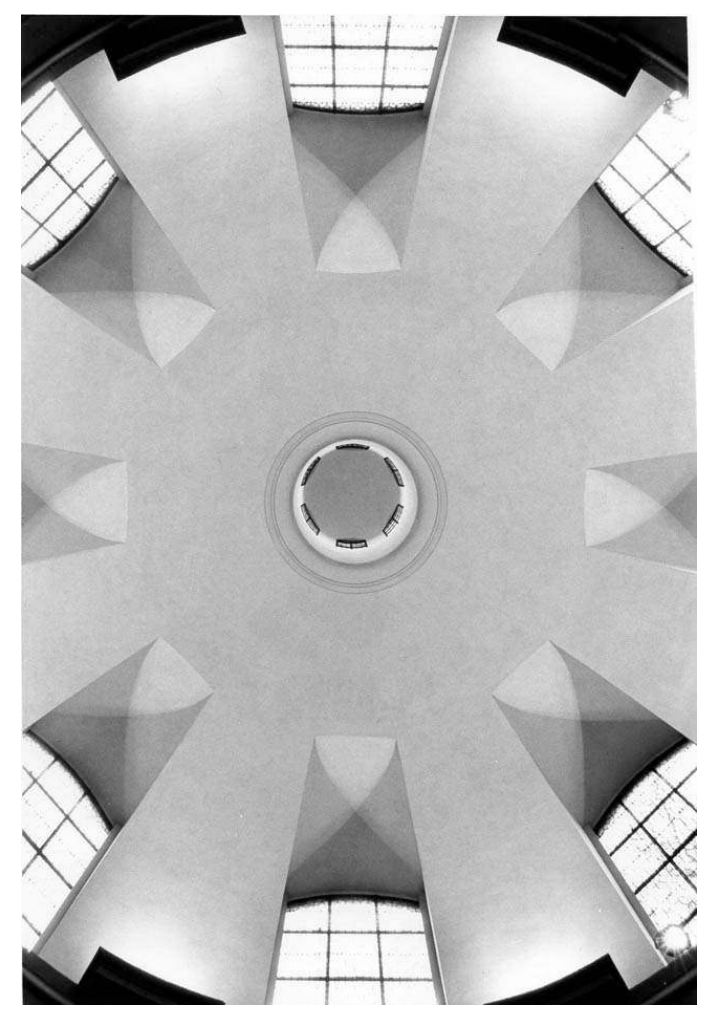

L'amphithéâtre de l'École de chirurgie, rue de l'École de médecine. La coupole de l'amphithéâtre. Photo. Jean-Christophe Doërr.

(c) Jean-Christophe Doërr.

Le nouvel édifice, dont la construction est confiée à Charles Joubert, est financé cette foisci grâce à un emprunt de 10000 livres. En dépit de difficultés pécuniaires, les travaux sont menés à bien en quatre ans. Le coup est terrible pour les médecins, bien incapables à la même époque de se lancer dans pareil projet... Tout au plus peut-on imaginer, comme le suggère François Fossier ${ }^{30}$, que c'est à cette époque qu'ils font dresser par l'agence de Jules Hardouin-Mansart les plans d'une « école de médecine ", aujourd'hui conservés dans le fonds Robert de Cotte de la Bibliothèque nationale de France. En l'absence de moyens, on peut penser que ces plans tiennent lieu de consolation, un rêve de faculté pour une institution dans la gêne... C'est en 1742, soit un demi-siècle après les chirurgiens, que la faculté de médecine, contrainte de mettre à terre l'amphithéâtre de Riolan rongé par les crues de la Seine, fait enfin bâtir un nouveau théâtre de dissection. Pour que l'architecte Barbier de Blignères puisse mener à bien son projet, ils décident de financer la construction en retenant la moitié des droits de licence et des actes ${ }^{31}$. Le 18 février 1745, il est inauguré en grande pompe, avec une leçon anatomique du Danois Jacques Bénigne Winslow. Son nom restera attaché au lieu plus longtemps que la faculté. (fig. $\mathbf{n}^{\circ} \mathbf{6 , 7 , 8 )}$ 


\section{Figure 6}

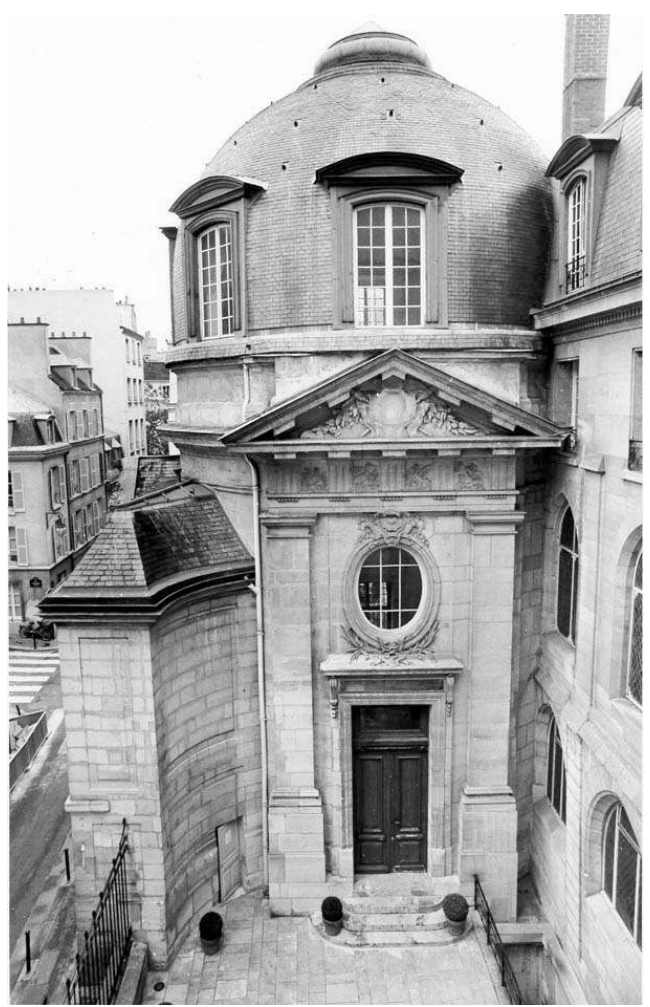

L'amphithéâtre de l'ancienne faculté de médecine de l'Université de Paris, rue de la Bûcherie. La façade principale. Photo. Jean-Christophe Doërr.

(c) Jean-Christophe Doërr. 
Figure 7

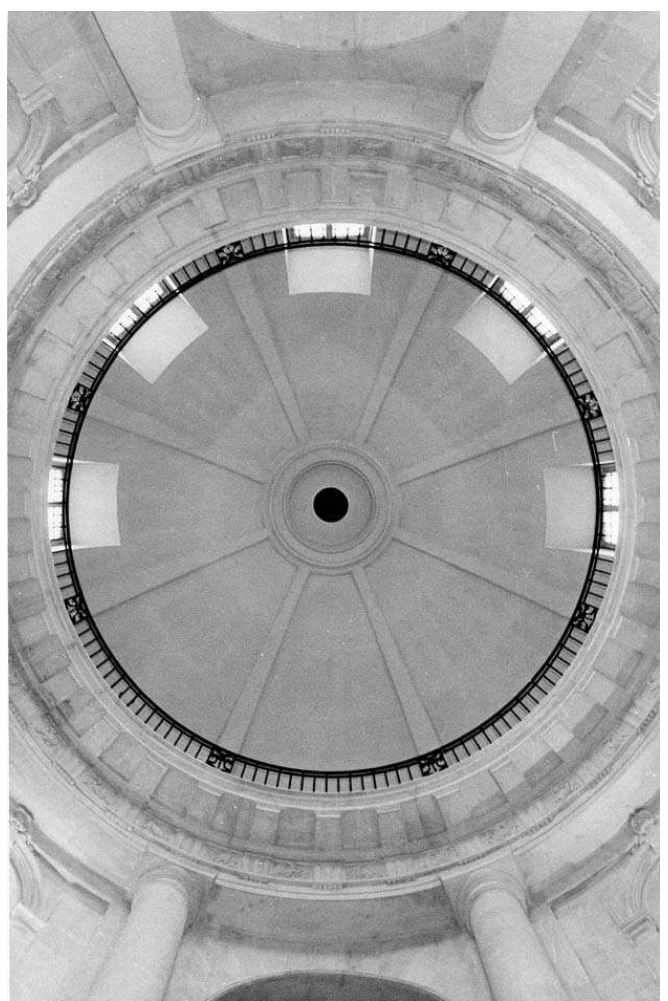

L'amphithéâtre de l'ancienne faculté de médecine de l'Université de Paris, rue de la Bûcherie. La coupole de l'amphithéâtre. Photo. Jean-Christophe Doërr.

(c) Jean-Christophe Doërr.

Le troisième acte entérine la victoire des chirurgiens. En 1775, la faculté de médecine, lassée de l'insalubrité du quartier, abandonne sans gloire la rue de la Bûcherie pour gagner au Clos-Bruneau les logis laissés vacants par les juristes ${ }^{32}$, installés depuis l'année précédente dans l'édifice conçu par Soufflot ${ }^{33}$. La même année, le 27 avril, Pichault de la Martinière inaugure la première séance publique tenue par l'Académie de chirurgie dans son nouveau collège, qui est, en fait, en véritable palais. 


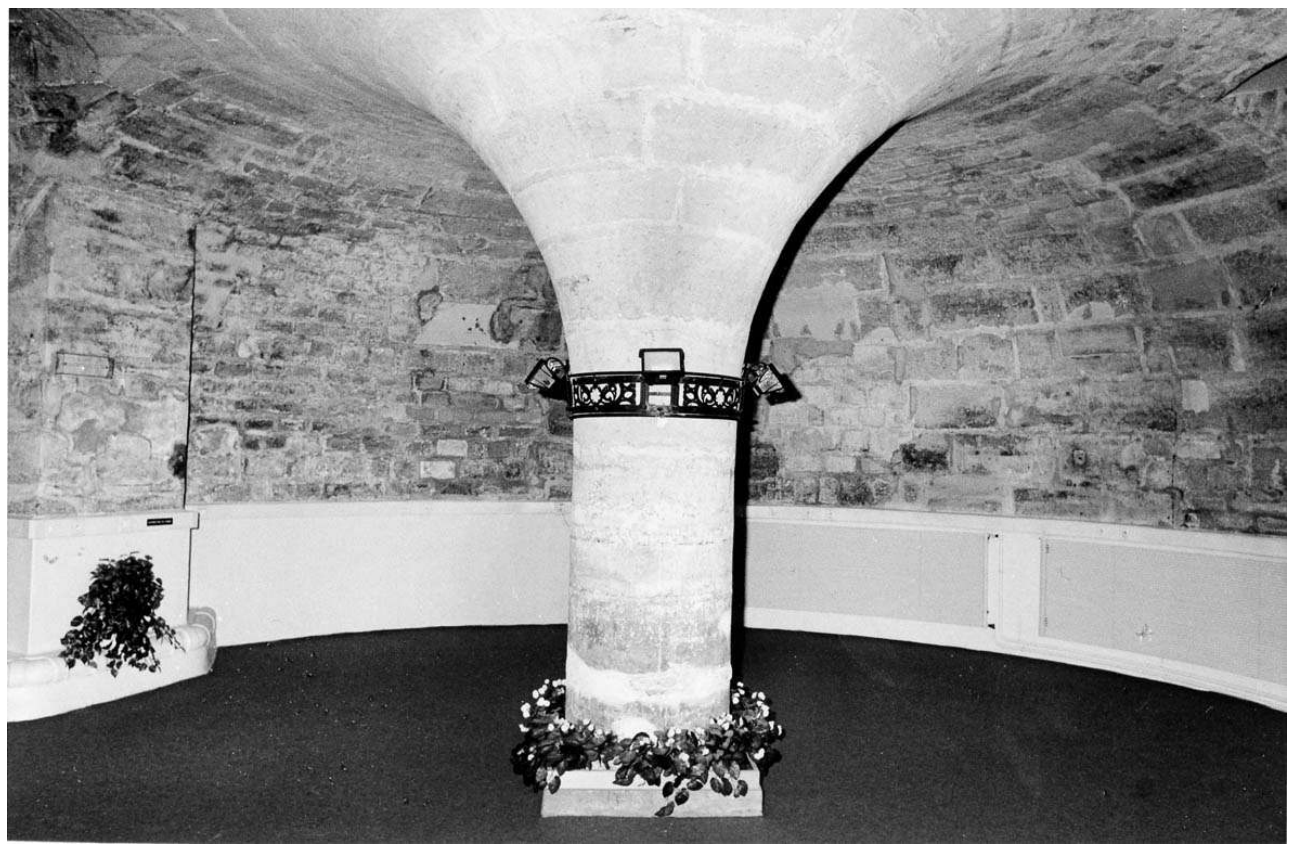

L'amphithéâtre de l'ancienne faculté de médecine de l'Université de Paris, rue de la Bûcherie. La cave située sous l'amphithéâtre. Photo. Jean-Christophe Doërr.

(c) Jean-Christophe Doërr.

\section{Technique et symbolique des amphithéâtres}

\section{Des dispositifs efficaces pour l'enseignement?}

Contrairement à l'amphithéâtre de Padoue, les réalisations parisiennes de l'époque moderne sont largement éclairées, afin d'assurer une excellente visibilité à l'assistance. Dans le cas de l'amphithéâtre des chirurgiens, trois niveaux de baies laissent pénétrer la lumière du jour : les six ouvertures du lanternon, les huit lucarnes du dôme et les œils-debœuf de l'attique. Le plan choisi, octogonal ${ }^{34}$, permet également une bonne répartition de l'auditoire, tandis que l'implantation du siège du démonstrateur, dos à l'entrée principale, évite à ce dernier de tourner le dos au public. On trouve un pareil souci de clarté dans l'œuvre de Barbier de Blignères, quoique ce parti demeure moins abouti du fait de la configuration des lieux. En effet, seules cinq grandes fenêtres sont percées dans le dôme, la proximité du bâtiment médiéval interdisant d'en ouvrir vers le midi. Sans doute a-t-on voulu corriger ce défaut en ouvrant entre la porte et le fronton triangulaire qui la surmonte un grand œil-de-bœuf, ce qui rend l'entrée principale étroite et resserrée. Surtout, on a ouvert au nord une très grande fenêtre en plein cintre qui donne directement sur la tribune surplombant la salle. Là encore, le dispositif paraît moins réfléchi, moins heureux que dans l'amphithéâtre des chirurgiens. Enfin, l'étroitesse de son diamètre, imposée par la configuration de la parcelle, achève de faire de l'amphithéâtre des médecins une copie de celui de Charles Joubert, mais une copie moins apte que l'original à remplir sa fonction ${ }^{35}$. Il représente pourtant pour la faculté une avancée incontestable; en effet, le projet mis au point par l'agence de Jules Hardouin- 
Mansart comportait certes un "amphithéâtre » implanté en face de la chapelle, mais celui-ci, de plan rectangulaire, aurait difficilement rempli sa fonction ${ }^{36 .}$

\section{Outils de représentation, objet de représentations, source d'imitations}

17 Morceau d'éloquence architecturale, l'amphithéâtre est aussi pour la communauté qui l'édifie prétexte à l'éloquence décorative, peinte ou sculptée, à moins qu'on ne préfère user de l'antique prestige de l'épigraphie. Comparer brièvement les procédés mis en œuvre par les uns et les autres éclaire certains aspects des représentations de chaque institution. C'est chez les chirurgiens que le décor est le plus parlant. Dès l'abord, le portail extérieur apparaît rehaussé de deux atlantes ailés portant trompette. Qu'on gagne la cour, et la façade principale, avec sa porte encadrée de colonnes ioniques, offre tout un discours à « la gloire renaissante de la chirurgie ». On y célèbre la construction de l'édifice à grand renfort d'inscriptions latines et françaises et de symboles (le coq d'Esculape, les boîtes des chirurgiens, et la couronne de France, indice des faveurs royales accordées à la compagnie). À l'intérieur, vingt-quatre portraits établissent « une véritable généalogie mythique de la communauté des chirurgiens de Paris depuis l'Égypte ancienne jusqu'aux nations contemporaines ${ }^{37}$, le tout sous le regard d'Apollon, ce qui constitue un nouvel hommage, à peine déguisé, à la personne royale. Placé en regard, l'amphithéâtre des médecins paraît bien plus sobre (fig. $\mathbf{n}^{\circ} \mathbf{9}, \mathbf{n}^{\circ} \mathbf{1 0}$ ). Sur les entablements extérieurs et intérieurs sont représentés les symboles de la médecine: coq, pélican, salamandre et cigogne. Mais si l'on cherche en vain l'évocation du pouvoir royal, c'est que la faculté peut se prévaloir de plus hautes autorités, spirituelles celles-là. Au fronton de l'entrée est reproduite la devise "Urbi et orbi salus", adoptée en 1460 à la suite d'une bulle de Nicolas $\mathrm{V}$ conférant aux licenciés de l'Université de Paris le droit d'enseigner en tout lieu sans examen préalable ${ }^{38}$. On peut toutefois trouver une autre explication à la modestie de ce décor. Contrairement aux chirurgiens, les médecins possèdent dans le bâtiment voisin une salle d'assemblée richement décorée, aux murs tendus de tapisserie relatant l'histoire de Psyché, à la cheminée barrée d'un grand Christ en croix, encore embellie par une importante collection de portraits des anciens maitres ${ }^{39}$; autant de signes d'une noblesse ancienne qui renvoient l'amphithéâtre de Saint-Côme et son décor au rang d'objet de luxe de parvenu. 
Figure 9

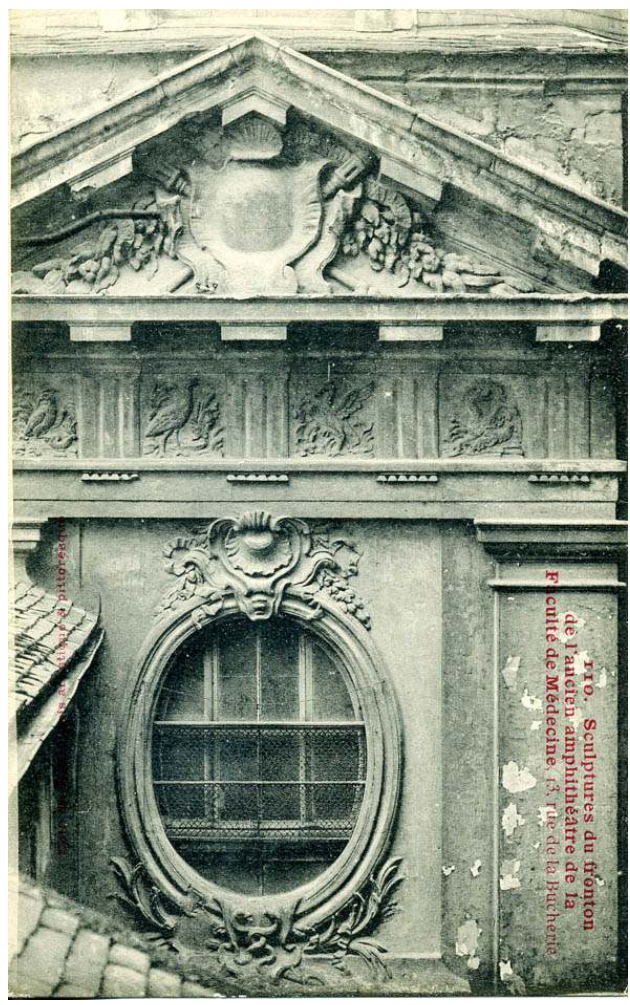

L'amphithéâtre de l'ancienne faculté de médecine de l'Université de Paris, rue de la Bûcherie. Le fronton de la porte d'entrée de l'amphithéâtre avant sa restauration. Carte postale ancienne. Collection du Vieux Paris Artistique et Pittoresque, $n^{\circ}$ 110. (collection particulière). 


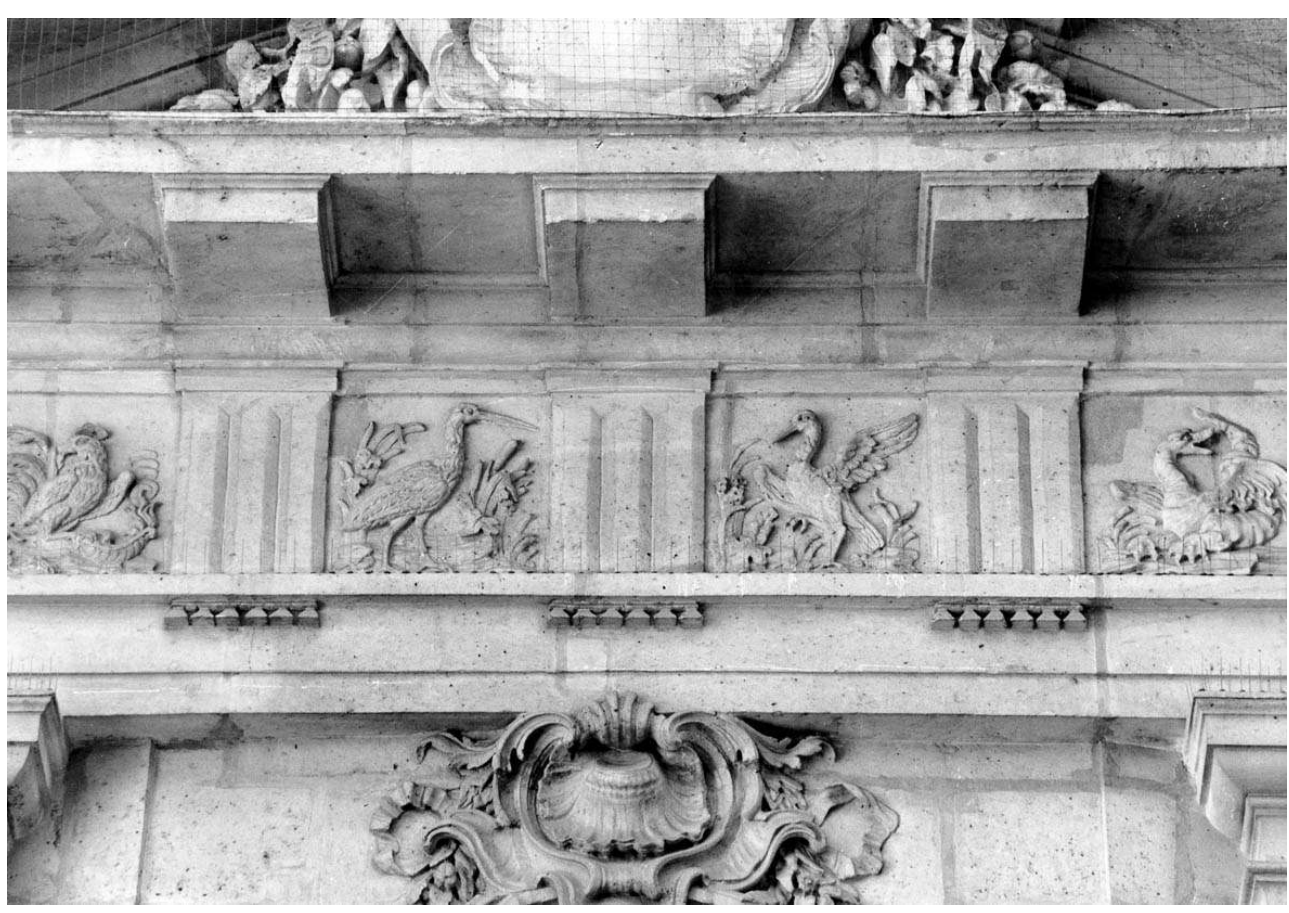

L'amphithéâtre de l'ancienne faculté de médecine de l'Université de Paris, rue de la Bûcherie. La frise de la porte d'entrée de l'amphithéâtre. Photo. Jean-Christophe Doërr.

(c) Jean-Christophe Doërr.

Ce dernier a pourtant eu, bien plus que l'amphithéâtre de Winslow, les honneurs des peintres et des graveurs, et ce d'abord à la demande des chirurgiens eux-mêmes, fiers d'exalter ce lieu de savoir qui est aussi une manifestation de pouvoir. L'un d'entre eux, Henri-Emmanuel Meurisse, fait graver une planche représentant le monument en élévation. Plus grand que nature, il apparaît au milieu des nuées, cerné d'allégories, écrasant de sa masse les rives de la Seine entre le Louvre et le collège des Quatre-Nations ${ }^{40}$ . D'autres, tel Pierre Dionis, le font reproduire à diverses reprises dans leurs ouvrages, en élévation ou en perspective intérieure ${ }^{41}$. Cinquante ans plus tard, Jean-François Blondel en donne une description précise, mais critique, agrémentée de planches ${ }^{42}$. Il n'est guère étonnant de constater qu'il sert par la suite de modèle pour les amphithéâtres de Bordeaux, Montpellier ${ }^{43}$, Perpignan, Louvain (en 1745) ou Toulouse ${ }^{44 .}$

\section{Conclusion}

Si l'amphithéâtre est un espace dont l'étude est essentielle pour comprendre le développement de l'architecture des établissements d'enseignement supérieur, il n'est toutefois pas le seul : salles de cours, d'études, bibliothèques, laboratoires font également partie de cet ensemble, alors que l'on observe le déclin progressif des espaces dédiés à l'habitat ou à la vie religieuse. Ces mutations sont particulièrement sensibles à partir de la deuxième moitié $\mathrm{du} \mathrm{XVIII}^{\mathrm{e}}$ siècle. Nouvelles techniques d'enseignement, nouvelles recherches architecturales, nouvelles formes de représentation des institutions, mais aussi expression des rivalités accrues entre elles: autant de traits qui annoncent les projets et réalisations des dernières décennies de l'Ancien Régime. En effet, cette époque 
est marquée non seulement par une profonde évolution stylistique et par une complexité croissante des types d'espaces dédiés à la transmission des connaissances, mais aussi par un nouveau contexte institutionnel : alors que l'Université de Paris peine à se réformer, de nouveaux établissements, les écoles spéciales, apparaissent. Elles bénéficient de la faveur du pouvoir royal, tout comme d'autres institutions d'enseignement (École de chirurgie, collège royal), plus anciennes mais elles aussi extérieures à l'Université. Commence alors une nouvelle phase de la rivalité entre médecins et chirurgiens, marquée par le triomphe des seconds sur les premiers, triomphe auquel Gondoin apportera sa manifestation la plus visible en concevant pour les chirurgiens une nouvelle école, appelée à devenir un modèle pour les architectures de l'enseignement supérieur... (fig. $\mathbf{n}$ - 11, no 12)

Figure 11

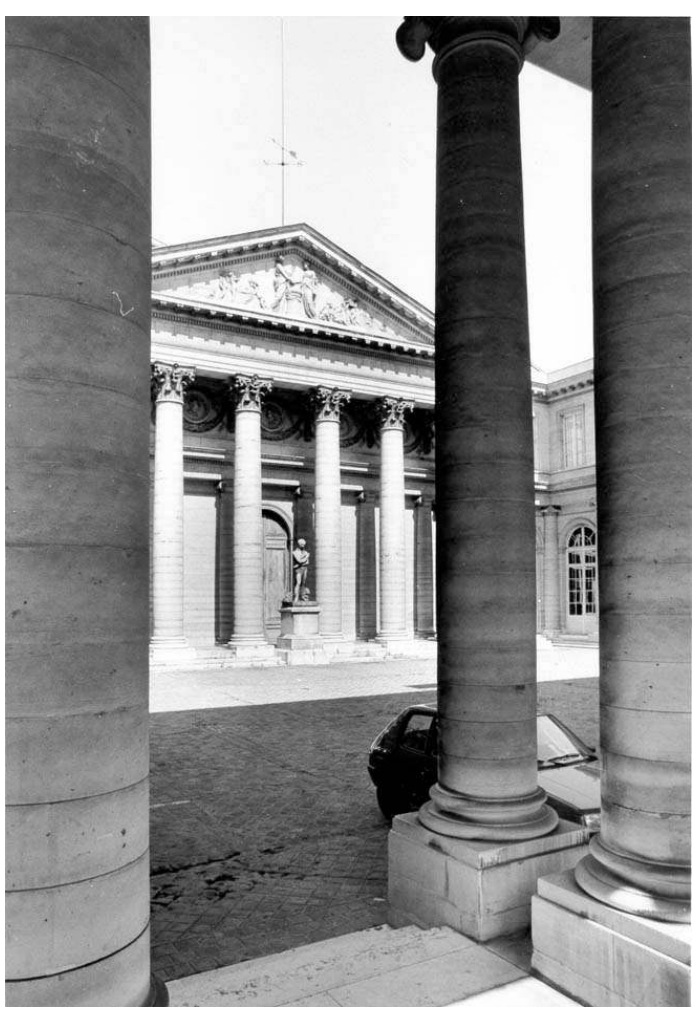

L'Académie de chirurgie, rue de l'École de médecine. La cour intérieure. Photo. Jean-Christophe Doërr. (c) Jean-Christophe Doërr. 
Figure 12

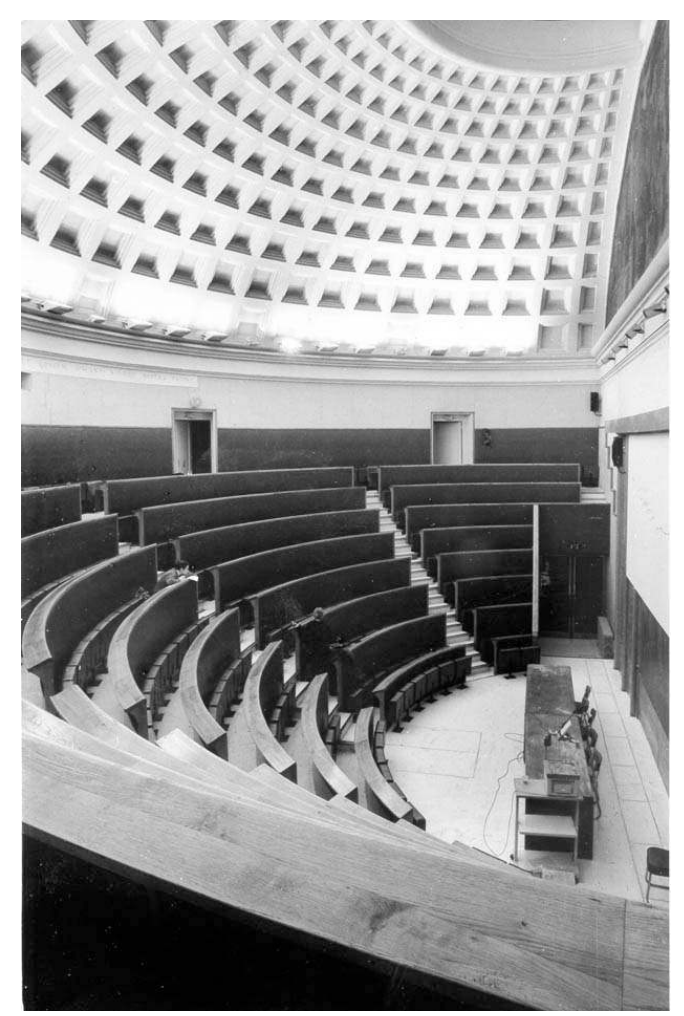

L'Académie de chirurgie, rue de l'École de médecine. L'amphithéâtre. Photo. Jean-Christophe Doërr. (C) Jean-Christophe Doërr.

\section{NOTES}

1. Les collèges des Bernardins, de Clermont (lycée Louis-le-Grand), de Bayeux, de Presles, de Beauvais, des Lombards, de Fortet, de l'Ave Maria, du cardinal Lemoine, des Écossais et des Irlandais ainsi que les séminaires des Trente-Trois et du Saint-Esprit. Subsistent en outre les bâtiments de la Nation de France.

2. ROUX, Simone. La rive gauche des Escholiers, Paris. Paris : Éditions Chrétiennes (coll. Vivre l'Histoire), 1992, p. 19.

3. MOULIN, Léo. La vie des étudiants au Moyen Âge. Paris : Albin Michel (Bibliothèque Albin Michel de l'Histoire), 1991, p. 212-213.

4. MOULIN, Léo. La vie des étudiants au Moyen Âge. Paris : Albin Michel (Bibliothèque Albin Michel de l'Histoire), 1991, p. 53-54.

5. D'IRSAY, Stephen. Histoire des université françaises et étrangères des origines à nos jours , T. 1. Paris : Picard, 1933, p. 153-156.

6. PEVSNER, Nikolaus. «Universities Yesterday ", The architectural review, vol. CXXII, $\mathrm{n}^{\circ} 729$, oct. 1957, p. 235-239. 
7. MOULIN, Léo. La vie des étudiants au Moyen Âge. Paris : Albin Michel (Bibliothèque Albin Michel de l'Histoire), 1991, p. 212. En 1432-1436, lors de la création de l'université, la ville multiplie les démarches pour que des locaux soient mis à la disposition de la nouvelle institution : requête au roi pour le prêt de la grande salle du château, au chapitre cathédral pour l'installation d'une librairie dans la chapelle des apôtres, paiement aux ordres mendiants d'un loyer pour l'occupation d'un local. La municipalité fournit en outre les bancs, chaises et pupitres nécessaires aux cours.

8. RÜCKBROD, Konrad. Universität und Kollegium. Baugeschichte und Bautyp. Darmstadt : Wissenschaftliche Buchgesellschaft, 1977, X-189 p., pl. 6-9.

9. RÜCKBROD, Konrad. Universität und Kollegium. Baugeschichte und Bautyp. Darmstadt : Wissenschaftliche Buchgesellschaft, 1977, X-189 p., pl. 7.

10. Le bilan de l'enquête générale sur les revenus de l'Université fait état en 1610 « d'une maison sise rue des Noyers, où pend pour enseigne la Madeleine, consistant en deux petits corps de logis, court et jardin, en laquelle se font ordinairement les congrégations et assemblées des députez de la dicte Faculté (...) ». Arch. nat., P 7731, déclaration de revenu annuel des théologiens de Paris, 8 février 1610. Octave Gréard ajoute en commentaire : «L'indication relative aux assemblées n'est qu'une satisfaction d'amour propre que la Faculté se donne: tous les textes la contredisent formellement ». GRÉARD, Octave. Nos adieux à la vieille Sorbonne. Paris : Hachette, 1894, p. 88, n. 1 .

11. ROUX, Simone. La rive gauche des Escholiers, Paris. Paris : Éditions Chrétiennes (coll. Vivre l'Histoire), 1992, p. 18.

12. FINOT, Docteur A. «La Faculté de Médecine de la rue Jean de Beauvais et ses doyens (1775-1793) ", La Montagne Sainte-Geneviève et ses abords, n 145, mai 1971, p. 51-60, p. 53.

13. Voir : LEGRAND, Noé. «L'Amphithéâtre de l'ancienne Faculté de Médecine et les jetons des doyens", Bulletin de la Montagne Sainte-Geneviève et de ses abords, t.3, 1899-1902, p. 182-195. Et NOIR, J. «L'Amphithéâtre de Winslow à la vieille Faculté de Médecine de Paris », La Montagne Sainte-Geneviève et ses abords, t. 7, 1920-1938, p. 200-203.

14. Après avoir accueilli la Maison des étudiants de l'Université de Paris, les bâtiments sont occupés par des services de la Ville de Paris.

15. PERRIN, Jules. «Les anciennes Écoles de Médecine de la rue de la Bûcherie et la Maison des Étudiants ", L'Architecture, 25 juillet 1921, p. 3-8, p. 3.

16. PERRIN, Jules. «Les anciennes Écoles de Médecine de la rue de la Bûcherie et la Maison des Étudiants ", L'Architecture, 25 juillet 1921, p. 3-8, p. 8.

17. PERRIN, Jules. «Les anciennes Écoles de Médecine de la rue de la Bûcherie et la Maison des Étudiants ", L'Architecture, 25 juillet 1921, p. 3-8, p. 3.

18. Pour synthèse sur les amphithéâtres, voir: HOTTIN, Christian. "L'Enseignement: les amphithéâtres ", Universités et grandes écoles à Paris. Les palais de la science, Paris, DAAVP, 1999, p. 45-51.

19. LENGLART, Denis, VINCE, Agnès. «Universités et grandes écoles ", Le Moniteur, Paris, 1992, p. 104.

20. PERRIN, Jules. «Les anciennes Écoles de Médecine de la rue de la Bûcherie et la Maison des Étudiants ", L'Architecture, 25 juillet 1921, p. 3-8, p. 4.

21. VÉSALE, André. De corporis humani fabrica. Bâle, 1543, p. 548, note a.

22. LAGET, Pierre-Louis. «Le premier amphithéâtre d'anatomie de la communauté des chirurgiens de Paris », Chirurgie, 1999, n 124, p. 681-690, p. 686. Ce texte de Pierre-Louis Laget, extrait de sa thèse de doctorat de Médecine ("Histoire des amphithéâtres d'anatomie: l'enseignement de l'anatomie à Paris du XVI ${ }^{\mathrm{e}}$ au XVIII ${ }^{\mathrm{e}}$ siècle », Paris, 1995) a également été publié dans le Bulletin Monumental, 1998, t. 156, IV, p. 369-384.

23. Ils ont été ainsi désignés pour les différencier des barbiers, ou « chirurgiens de robe courte ». 
24. Statuts, privilèges et règlements du collège de chirurgie de la ville de Paris, Paris, 1743, p. 112 .

25. PERRIN, Jules. «Les anciennes Écoles de Médecine de la rue de la Bûcherie et la Maison des Étudiants ", L'Architecture, 25 juillet 1921, p. 3-8, p. 5.

26. Statuts, privilèges et règlements du collège de chirurgie de la ville de Paris, Paris, 1743, p. 108.

27. Par une ironie de l'histoire, c'est sur l'emplacement de ce collège que les chirurgiens font élever entre 1770 et 1775 , grâce à la faveur royale et sur les plans de l'architecte Gondoin, leur nouvelle académie...

28. PERRIN, Jules. «Les anciennes Écoles de Médecine de la rue de la Bûcherie et la Maison des Étudiants ", L'Architecture, 25 juillet 1921, p. 3-8, p. 6.

29. Arch. nat., C.H.A.N., Min. centr. Carnot notaire. Étude XCI, liasse 484 (avril-juin 1691). Délibération prise lors de l'assemblée générale du 13 mars 1691. Cité par Pierre-Louis Laget, «Le premier amphithéâtre d'anatomie de la communauté des chirurgiens de Paris », Chirurgie, 1999, $\mathrm{n}^{\circ} 124$, p. 681-690., p. 683.

30. FOSSIER, François. Les dessins du fonds Robert de Cotte à la Bibliothèque nationale de France. Architecture et décor, Paris, Bibliothèque nationale de France - École française de Rome, Paris et Rome, 1997, p. 163-165.

31. PERRIN, Jules. «Les anciennes Écoles de Médecine de la rue de la Bûcherie et la Maison des Étudiants ", L'Architecture, 25 juillet 1921, p. 3-8, p. 7.

32. Les médecins continuent toutefois d'utiliser l'amphithéâtre de Winslow pour les leçons pratiques. Les grandes écoles de droit sont aménagées pour les cours et examens (rez-dechaussée), les assemblées (premier étage) et la bibliothèque (deuxième étage). FINOT, Docteur A. «La Faculté de Médecine de la rue Jean de Beauvais et ses doyens (1775-1793) », La Montagne Sainte-Geneviève et ses abords, $n^{\circ}$ 145, mai 1971, p. 51-60, p. 55.

33. Selon Pierre Poncelin, l'emplacement faisant face à la faculté de droit aurait pu être réservé pour la faculté de médecine, et non pour le siège de l'Université : «S'il est vrai, comme on l'assure, que l'intention du ministère soit de faire bâtir des écoles, sur la place Sainte-Geneviève, en face de celle de Droit, cette générosité, vraiment digne d'une administration éclairée, donnera un nouvel éclat à la Faculté ». PONCELIN, Pierre. Histoire de Paris, et description de ses plus beaux monuments, Dessinés et gravés en taille douce par F.N. Martinet, ingénieur, dessinateur et graveur du cabinet du roi, t. III, Paris, 1781 , XI-420 p., p. 81.

34. Cette configuration pourrait avoir été inspirée par l'amphithéâtre d'anatomie de Londres, bâti en 1672 par Robert Hook. LAGET, Pierre-Louis. « Le premier amphithéâtre d'anatomie de la communauté des chirurgiens de Paris », Chirurgie, 1999, n 124, p. 681-690, p. 686.

35. Il faut en revanche souligner les qualités architecturales et architectoniques intrinsèques de ce monument, en particulier la stéréotomie de la salle circulaire située sous l'amphithéâtre, dont la voûte est supportée par un pilier central se déployant en palmier.

36. B.n.F, Est., Va 260, R de C. 463 (microfilm H 41734). Reproduction : FOSSIER, François. Les dessins du fonds Robert de Cotte à la Bibliothèque nationale de France. Architecture et décor. Paris : Bibliothèque nationale de France - École française de Rome, Paris et Rome, 1997, p. 164.

37. LAGET, Pierre-Louis. «Le premier amphithéâtre d'anatomie de la communauté des chirurgiens de Paris », Chirurgie, 1999, n 124, p. 681-690, p. 688.

38. PERRIN, Jules. «Les anciennes Écoles de Médecine de la rue de la Bûcherie et la Maison des Étudiants ", L'Architecture, 25 juillet 1921, p. 3-8, p. 8.

39. PERRIN, Jules. «Les anciennes Écoles de Médecine de la rue de la Bûcherie et la Maison des Étudiants ", L'Architecture, 25 juillet 1921, p. 3-8, p. 8. 
40. MEURISSE, Henri-Emmanuel. Explication de l'estampe qui représente le profil de l'amphithéâtre anatomique que la compagnie des Mes chirurgiens jurés de Paris a fait nouvellement construire. Paris, 1694, $16 \mathrm{p}$.

41. DIONIS, Pierre. L'anatomie de l'homme suivant la circulation du sang et les dernières découvertes. Paris, 1694, 1re planche.

42. BLONDEL, Jean-François. Architecture française. Paris, 1752-1756, t. II, chapitre XV, pl. 1.

43. L'amphithéâtre de Saint-Côme de Montpellier, élevé entre 1752 et 1757 grâce à un legs de 100000 livres de La Peyronie surpasse pourtant largement son modèle parisien. Selon Giral, « il y a un [amphithéâtre de] Saint-Cosme à Paris, mais ce n'est pas un modèle à suivre. Il faloit imaginer et inventer, il faloit du nouveau ». De fait, l'édifice est beaucoup moins affaissé, écrasé par son dôme que celui de Paris (c'était aussi la principale critique de Blondel), ses proportions sont plus élancées, le corinthien est employé partout. Son rez-de-chaussée, traité en portique, permet d'apercevoir la salle depuis la rue. VAYSSETTES, Jean-Louis, SOURNIA, Bernard. "L'amphithéâtre de Saint-Cosme», Le Guide du patrimoine. Languedoc-Roussillon (JeanMarie Pérouse de Montclos, dir.). Paris : Hachette, 1996, p. 319-320.

44. LAGET, Pierre-Louis. "Le premier amphithéâtre d'anatomie de la communauté des chirurgiens de Paris », Chirurgie, 1999, n 124, p. 681-690, p. 689.

\section{RÉSUMÉS}

On étudie ici les conditions d'émergence, à partir de la fin du Moyen Age, des espaces spécifiques à la transmission du savoir dans l'espace parisien. Si les collèges sont les lieux les plus emblématiques de l'existence de l'Université de Paris, apparaissent aussi des édifices dévolus à l'accueil des facultés et des écoles. C'est en leur sein, et plus particulièrement dans les communautés formées par les médecins et les chirurgiens, qu'apparaissent les premiers amphithéâtres, imités des réalisations italiennes antérieures. Tout au long du XVII ${ }^{\mathrm{e}}$ et du XVIII ${ }^{\mathrm{e}}$ siècles, la construction de ces édifices revêt un double caractère : perfectionnement technique d'une part, mais aussi et surtout enjeu et manifestation d'une rivalité constante entre les deux corporations. Plusieurs de ces édifices existent encore aujourd'hui. Ils méritent d'être considérés en raison de leur qualité architecturale, mais encore en tant que témoignages de ces luttes institutionnelles.

This article studies the emergence, from the end of the Middle Ages, of specific spaces in Paris devoted to the transmission of knowledge. Colleges are perhaps the most characteristic places making up the university of Paris, but other buildings also appeared for individual faculties and schools. It was here, and in particular in the communities formed by doctors and surgeons, that the first amphitheatres, or lecture halls, appeared, inspired by Italian models. Throughout the seventeenth and eighteenth centuries, the construction of these buildings had a dual character : on the one hand they demonstrated increasing technical perfection, but at the same time they were also expressions of the rivalry between the two corporations. Several of these places still exist today and deserve more attention for their architectural interest and for the traces they preserve of these institutional conflicts. 
INDEX

Mots-clés : Barbier de Blignères, Charles Joubert, Ecole de chirurgie, Faculté de médecine, Gondoin, Henri-Emmanuel Meurisse, Jacques Bénigne Winslow, Jean-François Blondel, Jules Hardouin-Mansart, Pichault de la Martinière, Pierre Dionis, Quartier Latin, Université de Paris, amphithéâtre

Keywords : Latin quarter, Lecture hall, Medical Faculty, Surgical school, University of Paris

\section{AUTEUR}

\section{CHRISTIAN HOTTIN}

Conservateur du patrimoine, chef de la Mission ethnologie, ministère de la Culture et de la Communication. christian.hottin@culture.gouv.fr 